Int. J. Speleol. 18 3-4 (1989), pp. 73-86

\title{
CARATTERI GENERALI DELLE MORFOLOGIE NELLE ALPI E PREALPI GIULIE E NEL CARSO TRIESTINO॰
}

\author{
Franco Vaia *
}

\section{PREMESSA}

La regione Friuli-Venezia Giulia può essere immaginata, per sommi capi, come una successione, degradante dal confine di Stato al Mare Adriatico, di balze decrescenti in quota e disposte per lo più secondo i paralleli. Come è noto esse sono il frutto dei piegamenti e delle dislocazioni subite dalle masse rocciose, oltre che delle variazioni di composizione litologica.

In altre parole, da Nord a Sud si susseguono rilievi di età sempre più recente, meno evoluti e meno resistenti. In realtà nei tempi geologici si assiste all'alternarsi di depositi da cui derivano litologie più o meno degradabili: pertanto, in ciascuna delle fasce di rilievi, cioè in ogni singolo blocco in cui si è scomposta la pila dei litotipi, si verifica in piccolo quanto osservato in generale lungo la sezioni meridiana della Regione.

L'arricciatura e la frammentazione dell'originaria coltre prevalentemente sedimentaria, litificata da processi molteplici, ha assunto uno schema ben preciso in funzione del verso delle spinte orogenetiche: gli assi delle strutture principali ed i piani principali di discontinuità tendono perciò ad essere disposti secondo le direzioni E-W e NW-SE, influenzati soprattutto dall'ultimo ciclo orogenetico e in particolare dalla ripresa della fase dinarica nel momento di acme.

Il sistema delle Alpi Giulie rappresenta una parte dell'intero complesso di rilievi esistenti entro i confini regionali, in quanto segue sia in senso meridiano che in senso parallelo alla catena carnica, che grosso modo coincide con gran parte del confine italo-austriaco.

Secondo alcuni Autori, la divisione tra i due sistemi corrisponde al meridiano della Val d'Aupa; tuttavia alcune strutture e formazioni si prolungano anche oltre questo confine, definito in sostanza da una Nord-Sud forse di non elevato ruolo geodinamico. Più esattamente dunque il com-

\footnotetext{
* Istituto di Geologia e Paleontologia, Università di Trieste.

- Lavoro eseguito con il finanziamento M.P.I. 60\% (resp. F. Vaia).
} 
plesso delle Alpi e Prealpi Giulie inizia in sinistra Fella e si svolge tutto in sinistra Tagliamento, fino alla pianura orientale e al Carso, che ne rappresenta in qualche modo una propaggine. Per quanto discutibile tale affermazione possa essere, l'anticlinale asimmetrica che conclude a Trieste la serie dei rilievi della porzione orientale della Regione si accosta molto a quelle strutture che in Friuli sono state definite "ellissoidi cretacici" e che bordano il limite con l'alta pianura friulana affiorando dalle formazioni flyschoidi deformate e sfondate da queste brachianticlinali carbonatiche.

Non è difficile d'altro canto seguire lo sviluppo delle serie di pieghe più o meno fagliate e separate talora da grandi piani classificabili come sovrascorrimenti veri e propri. Dalle propaggini più settentrionali alla riva dell'Adriatico orientale, o meglio del Golfo di Trieste, queste strutture si svolgono in un insieme che, se non altro in apparenza, definisce un ordine stringato, un concatenarsi logico, per quanto complessi siano stati i momenti di formazione e di sviluppo.

I culmini principali delle Alpi Giulie sono i Monti di Dogna, i gruppi del Montasio e del Canin, per finire con il Mangart al confine con la Jugoslavia.

La parte prealpina vede invece emergere il M. Plauris, la catena dei Musi, quella dell'Ambruseit-Cjampòn e del Gran Monte e più a valle l'insieme flyschoide e degli ellissoidi, con i monti Matajùr, Mia, Lùbia, Bernàdia, e Faeit-Campeòn.

In senso parallelo le età geologiche sono analoghe, essendo prevalenti nelle fasce alpine giulie le formazioni triassiche e giurassiche (in subordine), con minori presenze permiane o cretaciche; nella parte prealpina si ripetono queste componenti, cui seguono a meridione le successioni tardocretaciche e cenozoiche prevalentemente clastiche. In mezzo ad esse, come detto, emergono i rilievi carbonatici cretacici.

\section{LE ALPI GIULIE}

Il portamento delle Alpi Giulie, rispetto alle Carniche e alle Tolmezzine (che sono la prosecuzione a Sud di queste ultime nella parte occidentale della Regione), è visibilmente diverso. Molti geografi e geologi, ma anche solo viaggiatori attenti, colsero e colgono tuttora le differenze morfologiche che caratterizzano i due gruppi.

Anzitutto premettiamo che non si tratta di quote, poiché è proprio nelle Carniche che il M. Cogliàns raggiunge la massima altitudine del Friuli-Venezia Giulia, anche se poi i due gruppi che seguono nella classifica appartengono alle Giulie (Canin e Montasio). 


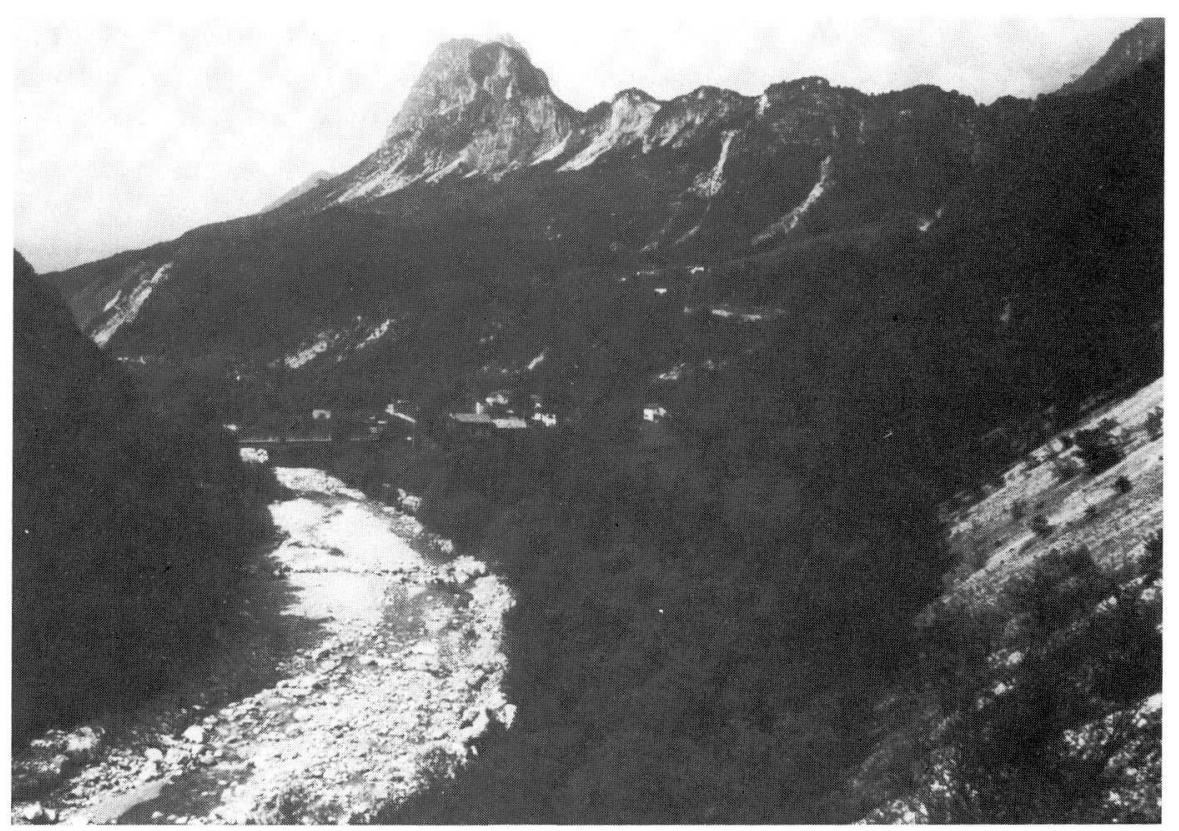

Fig. 1 - Solco della Val d'Aupa (Nord-Sud) con la dorsale glacializzata del M. Vualt (Alpi Giulie). Si notino le cospicue fasce detritiche e le frequenti forme franose sui ripidi versanti.

Aupa valley (North-South directed, Julian Alps). The Vualt Mt ridge appears on the right side, with clear glacial modelling. We notice the plentiful debris and landslides on the mountain sides.

Si tratta invece di forme connesse evidentemente soprattutto con la litologia.

Il richiamo alle Dolomiti trentine e bellunesi è spesso evidente. Man mano che fin dalla base dei rilievi affiorano le formazioni carbonatiche tipiche (calcari, calcari dolomitici e dolomie), il paesaggio muta da occidente a oriente e da Nord a Sud facendo di questo areale giulio un'entità quasi completamente a sè stante.

A ciò contribuiscono in varia misura i caratteri del sistema di discontinuità (giaciture, frequenze, estensioni lineari e areali) siano esse semplici diaclasi oppure faglie o sovrascorrimenti.

Compaiono allora ampii piastroni incisi da strette e aspre vallecole, alti sui fondivalle a formare pianori di vetta o prolungati verso le sommità da torrioni e bastionate in variabilissima successione orizzontale.

Poco frequenti le valli e pertanto le acque di superficie, imponenti le pareti con elevati dislivelli. 
La porzione orientale della regione, con i suoi caratteri climatici, permette poi di conservare ancora le ultime tracce della glaciazione, con le vedrette residuali del Canin e del Montasio. Tracce infinitesime, ormai, ma importanti, poiché testimoniano il recente ritiro delle masse maggiori e si accompagnano a forme freschissime sia di esarazione che di accumulo.

Il fatto che le aste che costituiscono la rete drenante siano per la gran parte strettamente condizionate, nella loro impostazione e successivo sviluppo, dall'esistenza del già citato sistema di discontinuità, tenendo conto del ruolo diverso sostenuto dalle diverse serie (in funzione della loro orientazione rispetto allo schema deformativo), consente di definire il reticolo come "a graticcio", con i collettori principali in posizione susseguente. In realtà ciò potrebbe essere discusso a lungo, poiché alcune aste subordinate sono da considerarsi in realtà eredi dei primordiali collettori: il fatto che esse siano in posizione di conseguenza, o antecedenza, potrebbe far mutare la classificazione del reticolo forse verso una individuazione scientificamente più corretta dello stadio in cui esso si trova.

Un fatto è certo: la porzione giulia del reticolo stesso non può essere vista troppo staccata da quella propriamente carnica, poiché attualmente il

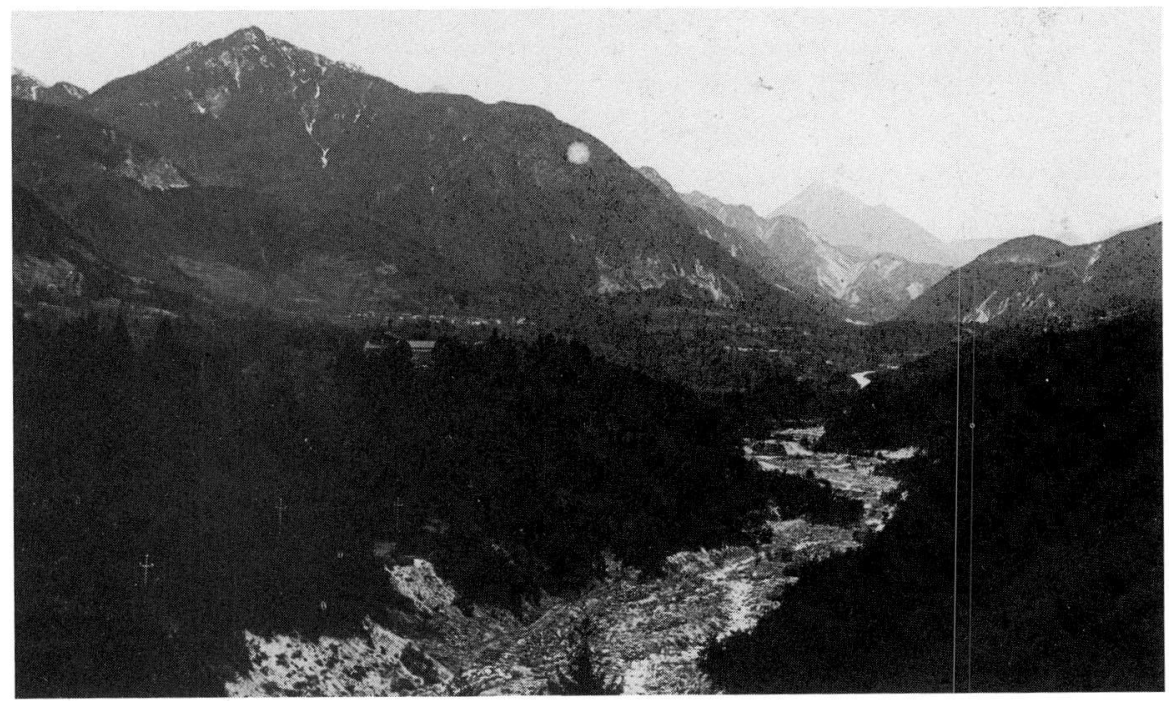

Fig. 2 - La media Val Resia (ENE-WSW) tra i gruppi montuosi del Canin (fuori campo a destra, Alpi Giulie) e dei Musi (a sinistra, Prealpi Giulie). Nel fondovalle i sedimenti quaternari terrazzati. The middle course of the Resia Valley (ENE-WSW directed) which lies between the Canin Mt (right, outside, Julian Alps) and the Musi Mts (left, Julian Prealps). Quaternary alluvial terraces appear at the bottom of the valley. 


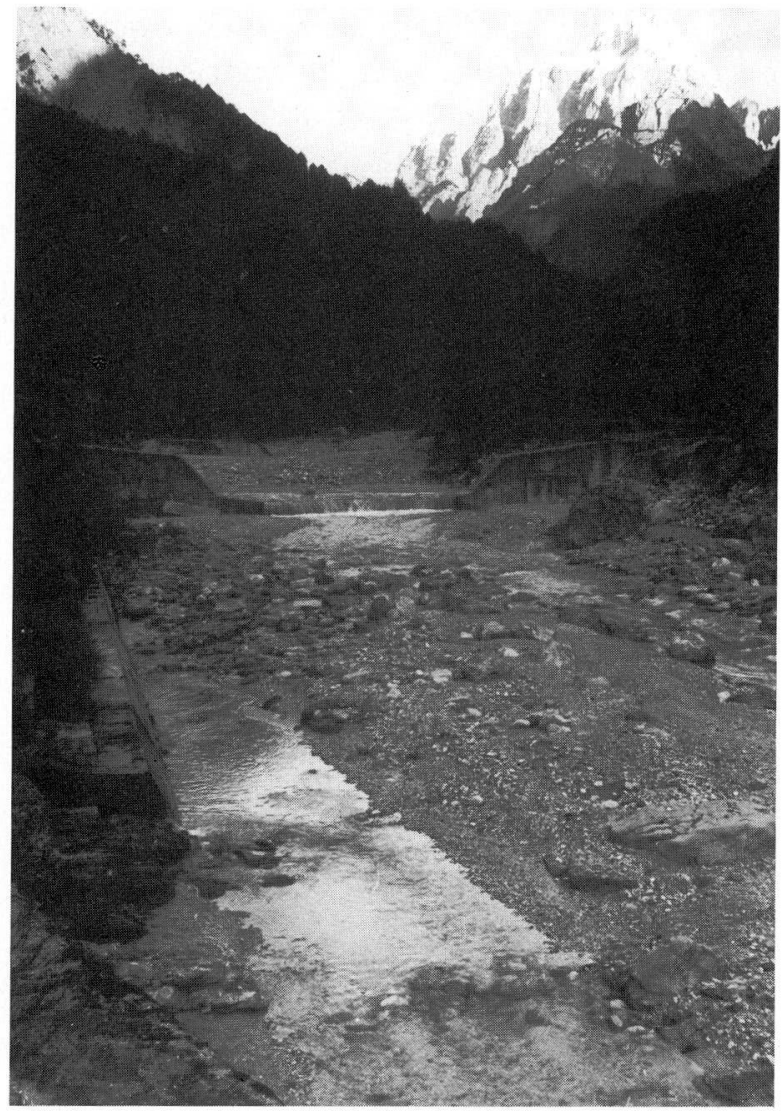

Fig. 3 - Difese contro l'intensa erosione esplicata dal Torrente Dogna (Est-Ovest) sulle formazioni triassiche tettonizzate. Sul fondo il massiccio del $\mathrm{M}$. Montasio (q. 2753 m.s.l.m.). Defence works against the high erosion of the Dogna River ( $E-W$ directed) on the tectonized triassic rocks. In the background the Montasio Mt $(9032$ $f t)$.

sistema appare come un tutt'uno a partire dallo spartiacque italoaustriaco. In quest'ottica risulta più "leggibile" il comportamento dei corsi d'acqua, soprattutto per quanto riguarda lo smaltimento del carico solido.

Se di norma infatti può apparire lineare il rapporto tra stato energetico (o potenziale erosivo) e carico solido, nel nostro caso molte anomalie sono state riscontrate, dovendosi in proposito ritenere, anche se non in maniera conclusiva, che esiste una sperequazione di comportamento tra i diversi settori in cui è suddivisibile la rete drenante regionale. In altre parole, pur nell'ambito di uno stadio del tutto simile in cui si trovano i diversi sottobacini, il convogliamento a valle del materiale prodotto e addirittura la produzione dello stesso appare diversa. Per tali motivi, la parte giulia risul- 
ta localmente più come un veicolo che non come fornitore importante di detrito (quindi di alluvioni) e spesso con tendenza a convogliare in media lentamente il materiale al di fuori dei singoli bacini.

Rimane tuttavia indiscutibile il fatto che l'effetto dei disturbi tettonici maggiori assieme ai valori per lo più elevati dell'acclività dei versanti sostiene naturalmente la rata di produzione. A ciò si aggiunga naturalmente l'effetto tuttora sensibilissimo delle vicende glaciali: i profili longitudinali della Val Fella, della Val Raccolana, della Val Resia e le loro diverse sezioni trasversali ne denunciano le conseguenze; le numerose forme rivelano la causa prima dei processi attualmente in atto e da cui tali forme derivano.

\section{LE PREALPI GIULIE}

Anche tra i due gruppi prealpini carnico e giulio si rileva una certa differenza, pur nell'ambito delle analogie strutturali, soprattutto nelle fasce più meridionali dove muta sostanzialmente la composizione litologica e l'orientazione degli assi.

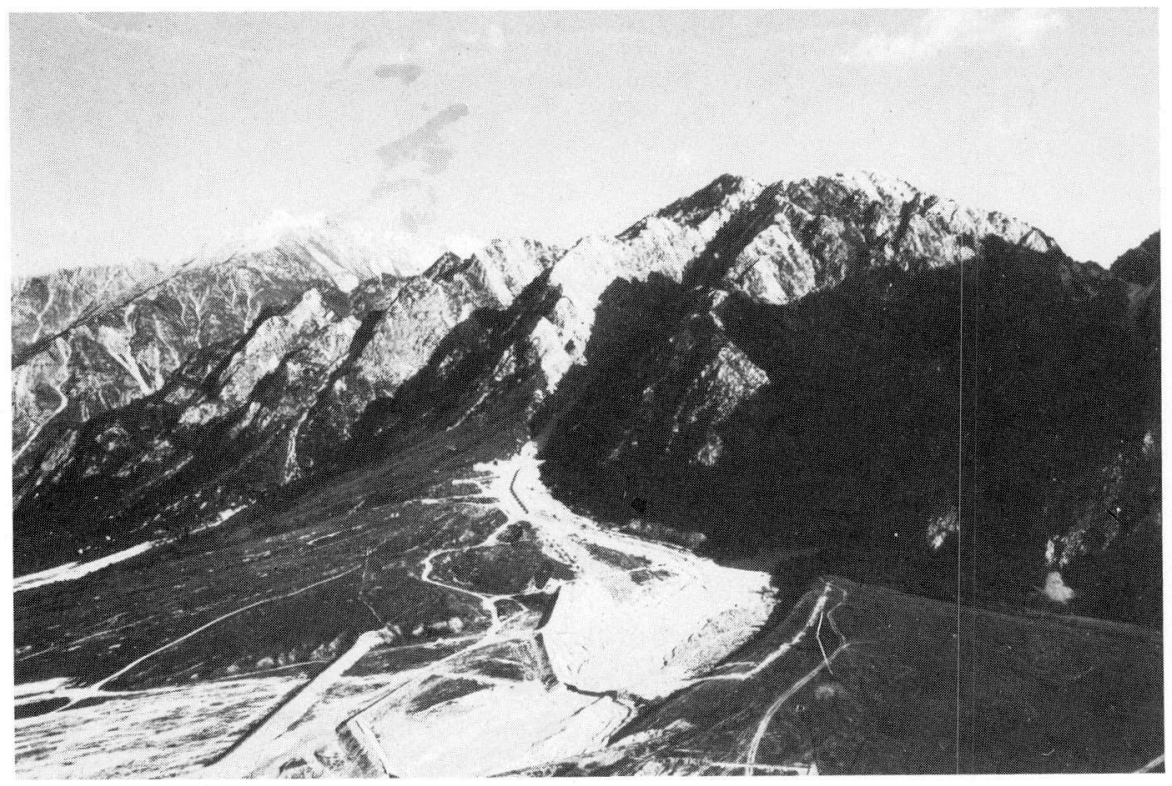

Fig. 4 - Il M. Ledis e la Vetta del Sole (Prealpi Giulie), fortemente tettonizzati, che alimentano i conoidi dei Rivoli Bianchi di Venzone, testimonianza dell'elevatissima attività erosiva in atto. The strongly tectonized Ledis Mt and Sun Peak (Julian Prealps), which feed the White Rills fans of Venzone. They point out the very high actual erosion. 


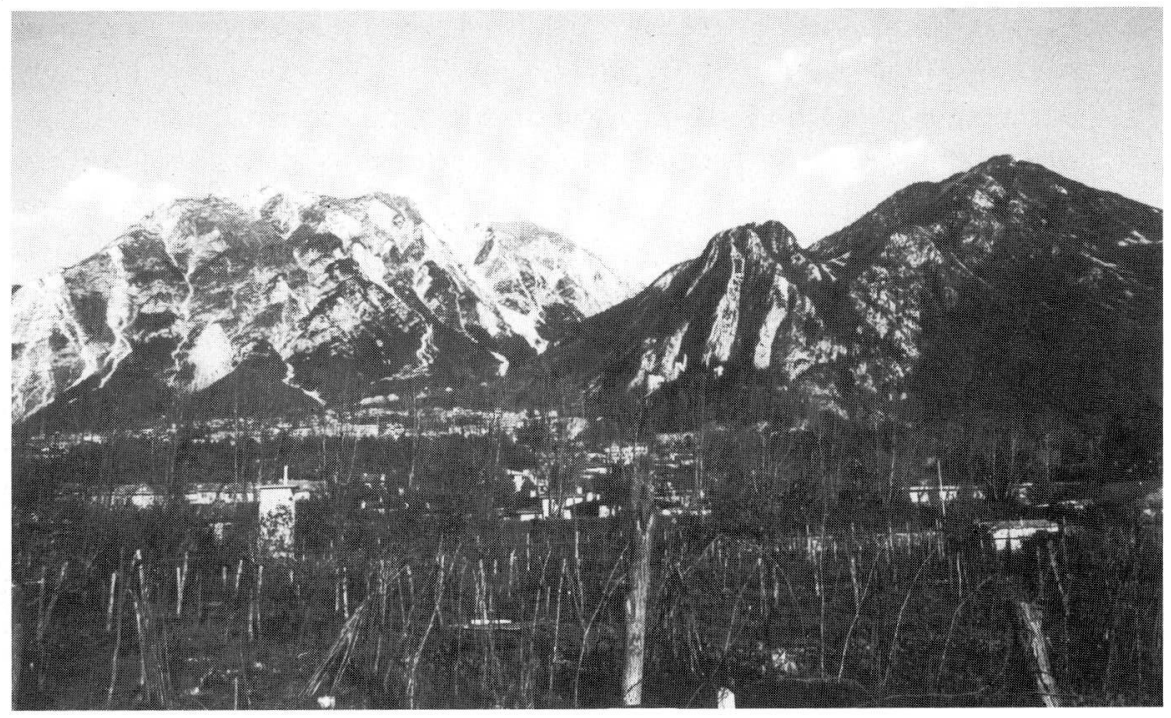

Fig. 5 - La catena di M. Cjampón, a sinistra, con le numerose frane da terremoto, e il M. Cuarnàn con il pilastro del Glemine, a destra, separati dal sovrascorrimento periadriatico orientato EstOvest (Prealpi Giulie). In primo e secondo piano il conoide di Gemona del Friuli, estesa costruzione alluvionale alimentata da un esiguo bacino.

The Cjampon chain, left, with its earthquacke landslides and the Cuarnàn Mt with the Glemine pillar, right, divided by the periadriatic overthrust $E-W$ directed (Julian Prealps). In the foreground lies the Gemona alluvial fan, fed by a very small drainage-basin.

Si osserva che a monte sono frequenti le similitudini morfologiche (tra alto Cellina, ad es., e alto Torre) mentre una brusca variazione di paesaggio avviene tra l'area in destra Tagliamento (M. Cavallo - M. Pala - M. Cuàr) prevalentemente carbonatica e quella in sinistra, tra Gemona e l'Isonzo, prevalentemente flyschoide. In altre parole nella parte prealpina orientale della regione spicca maggiormente la disomogeneità tra le due fasce ad andamento parallelo in cui essa si può suddividere: carbonatica la prima, clastica eterogenea la seconda. A Nord il M. Plàuris, il M. Musi, il M. Cjampòn ecc. si presentano con forti elevazioni e pendenze, con bastionate imponenti e ripide; a Sud il M. Faeit-Campeòn, lo Iàuer, lo Joannàz e via via i colli del Cividalese e del Goriziano degradano dolcemente verso la pianura con forme morbide e molto articolate, con possibilità di facile ed intenso smantellamento che ulteriormente differenzia, o meglio a tutt'oggi ha differenziato, il paesaggio. Naturalmente anche in questa fascia l'incrociarsi dei lineamenti ad andamento cosiddetto "alpino" con quelli ad andamento "dinarico" (rispettivamente assi E-W e NW-SE) in- 
fluisce sulla morfogenesi e pertanto sull'aspetto del paesaggio via via derivatone.

Tuttavia lo sviluppo della rete drenante mostra di subirne il condizionamento nella fascia settentrionale, con susseguenze evidenti e conseguenze altrettanto chiare; a meridione ciò non appare con immediatezza, almeno a grande scala, e in ogni modo ogni coniugazione di verso di deflusso con lineamento strutturale è molto più difficile da dimostrarsi con sicurezza.

Si rileva invece immediatamente la sostanziale variazione della densità di drenaggio nelle due fasce, a conferma dei diversi presupposti geologici. La stessa presenza d'acqua in superficie nel tempo conferma quanto illustrato poco sopra, anche sfrondando i regimi dal tributo dei sistemi carsici, che non solo interessano le successioni carbonatiche a settentrione, ma operano selezione idrica in numerose aree interessate dalla successione flyschoide, là dove si intercalano le potenti bancate calcarenitiche.

La rete sviluppatasi nella successione clastica, per quanto fittamente suddivisa, è infatti caratterizzata a lungo da scorrimento superficiale, sia pur con portate assai variabili in funzione degli afflussi (peraltro fino ad oggi decisamente abbondanti in questo settore, noto come il più piovoso d'Italia), mentre a monte l'assorbimento relativamente agevole asciuga gli alvei con rapidità.

Si osserva infine che il modellamento glaciale risulta ancora molto bene a settentrione: nell'alta valle del Torre, per portare un esempio tipico, sono chiari i terrazzi in roccia che interrompono la continuità delle pendenze del truogolo assai evidente, mentre le fasce inferiori dei versanti sono raccordate al fondovalle da estese coltri di copertura sciolta costituita da cospicui coni detritici e torrentizi poco evoluti e non ancora ben colonizzati.

A meridione qualche forma è stata definita glaciale e sugli altipiani flyschoidi placche sciolte sono attribuibili al till glaciale, ma in generale non si riscontra un paesaggio che possa essere chiaramente assimilato all'ambiente glacializzato, sia per la minor intensità del processo sia per la reazione successiva dei litotipi affioranti e già descritti.

\section{IL CARSO}

Riemergendo dalla copertura flyschoide che lo circonda a NW e a S, a meridione delle antiche strutture isontine oltre il solco del Vipacco, affiora il complesso cretacico che definisce il Carso. 
Come si è già detto, la struttura elementare è quella di una dolce anticlinale, che tuttavia, per la sua configurazione, si accosta agli "ellissoidi" friulani delle prealpi.

Tale assetto ha facilitato l'impostazione del processo carsico, qui particolarmente evoluto, in funzione dei valori di giacitura e in particolare di inclinazione della stratificazione.

È necessario però ricordare che la successione non è costituita esclusivamente da calcari puri e che essa, comunque, non presenta omogeneità all'interno delle singole formazioni. In altre parole, sia in senso verticale che in quello orizzontale la carsificabilità varia notevolmente. La sola osservazione di una carta topografica a media scala (ad esempio, quella 1:50.000 dell'I.G.M.) pone in luce in superficie fasce con forme epigee concentrate e allineate secondo la direzione media assiale della struttura e fasce con morfotipi carsici meno frequenti e in aree non continue.

In quest'area appaiono particolarmente evidenti gli influssi delle direttrici " dinariche" sulla morfogenesi; d'altro canto, questo è l'andamento dell'intera anticlinale. Tuttavia lineamenti di tipo "alpino" vengono in luce e si comportano come tali anche nella caso della carsogenesi.

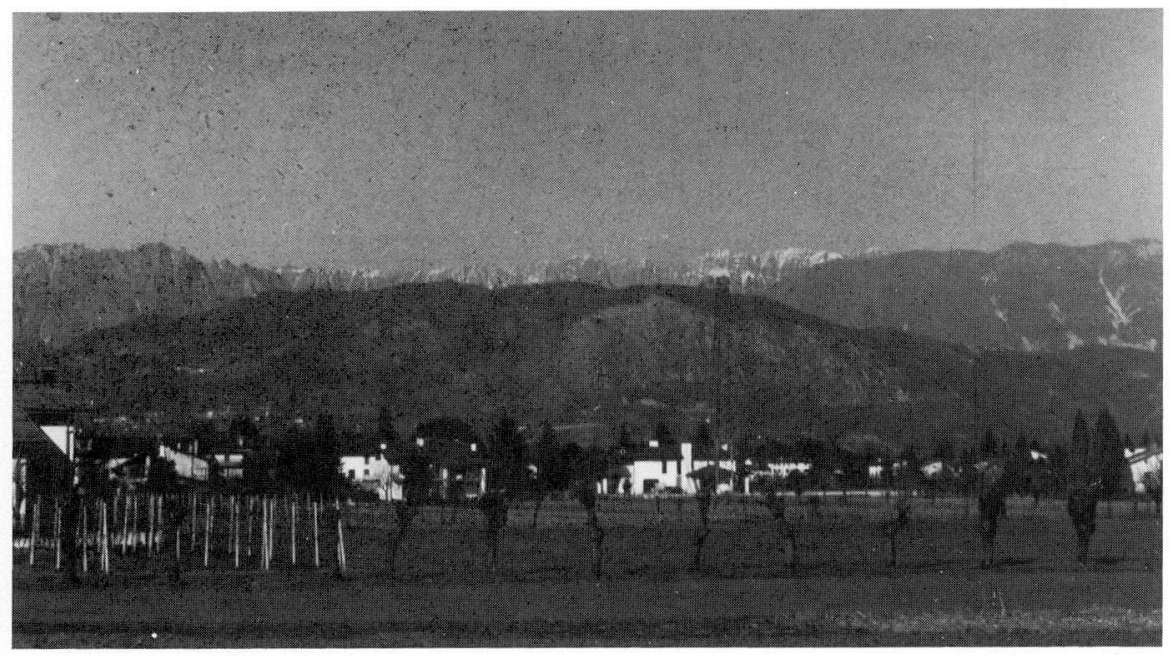

Fig. 6 - Le Prealpi Giulie al contatto con l'alta pianura friulana tra Tarcento e Nimis. In secondo piano l' "ellissoide" cretacico del M. La Bernardia. Sul fondo la catena dei Musi.

The boundary between Julian Prealps and the high friulian alluvial plain, near Tarcento and Nimis. In the foreground the cretacic "ellipsoid"' of the Bernardia Mt. In the background the Musi Mts chain. 
Nello stesso modo risaltano chiare le culminazioni al di sopra della quota media dell'altopiano, costituite dalle eteropie di tipo soprattutto dolomitico (ad esempio, da Rupingrande a Sgonico e Medeazza, fino al lago di Doberdò, in cui la dolomia costituisce il substrato al letto del sistema carsificato).

Analogo comportamento hanno le formazioni aptiano-albiane a calcari lastroidi bituminosi, dal M. Lanaro al M. Ermada e oltre, che sono soggette a manifestazioni di dissoluzione meno imponenti per quanto visibili e inseribili nell'ambito del Carso.

In breve, dal confine al bordo dell'altopiano si intuisce un susseguirsi di culminazioni e depressioni, ciascuna peraltro non omogenea in senso assiale, corrispondenti al diverso litotipo affiorante. Si osserva che nello stesso senso si procede dall' Aptiano al Luteziano.

Alle ondulazioni specificamente attribuibili alla diversa intensità del processo carsico, o meglio alla diversa carsificabilità delle formazioni, si affiancano i resti della rete fluviale antica (Valloni di Doberdò, di Brestovizza e di Aurisina, orientati tipicamente secondo gli assi "dinarici'); tuttavia si osserva che la originaria superficie di erosione miopliocenica doveva situarsi qualche decina di metri sopra l'attuale, prescindendo dalle vicende tettoniche recenti.

Nel contempo ai tipi morfologici epigei principali (le doline) e minori, ma spettacolari (gli insiemi di karren e di vaschette), si affiancano forme ipogee talora molto ampie (Grotta Gigante, Grotta di Trebiciano) e numerosissime. Tutto ciò articola enormemente le manifestazioni del processo dissolutivo, complicando ulteriormente il paesaggio, già movimentato dai presupposti citati.

Il Carso presenta però anche forme spettacolari che non sono carsiche: la Val Rosandra ne l'esempio più bello.

Accanto ai complessi industriali di Trieste esiste, tuttora incontaminato, un ambiente che ricorda più le Alpi che non il mare sciabordante a pochi passi. Il vero e proprio canyon che raccorda la piana paracostiera di Bagnoli con il confine italo-jugoslavo a Bottazzo testimonia ben altra dinamica, pur potendosi rilevare in esso tipi morfologici e idrogeologici del tutto dipendenti dalle manifestazioni carsiche in atto al contorno.

Il rilevamento geologico dà in qualche modo ragione di queste forme, poiché permette di riconoscere una successione litologica estremamente disturbata da ripetizioni in coincidenza di numerosi ed importanti piani tettonici di diverso carattere. Tutt'ora non si può ritenere che sia stata scritta l'ultima parola dell'interpretazione di quest'area, ma il soddisfacente pro- 
gresso delle indagini consente di migliorare la conoscenza del ruolo assunto da essa nel contesto strutturale del Carso e del raccordo tra Giulie e Dinaridi.

Ciò che interessa in questa sede è comunque la conseguenza degli eventi tettonici, espressa da bellissime forme di erosione accelerata, da episodi di evoluzione immediata dei versanti, da processi di colonizzazione faticosa da parte della vegetazione, da tratte d'alveo nette e spigolose, da terrazzi in vecchie alluvioni più o meno cementate (presso il Rifugio Premuda, alla fine della forra).

Ciò che ancor più colpisce, a questo proposito, è la quasi totale assenza di depositi attuali: gli alvei sono puliti o vi transitano elementi anche grossolani che non vi sostano a lungo. Un motivo in più per ritenere tuttora vivacissima la morfogenesi.

Fig. 7 - La forra della Val Rosandra (Carso Triestino), in un raro momento di siccità. La freschezza delle forme erosive denuncia la fase di dinamicità che coinvolge il Carso.

The Rosandra Valley gorge (Triest Karst) during an exceptional drought period. The erosional types youth shows the structural dynamism which involves the Karst.

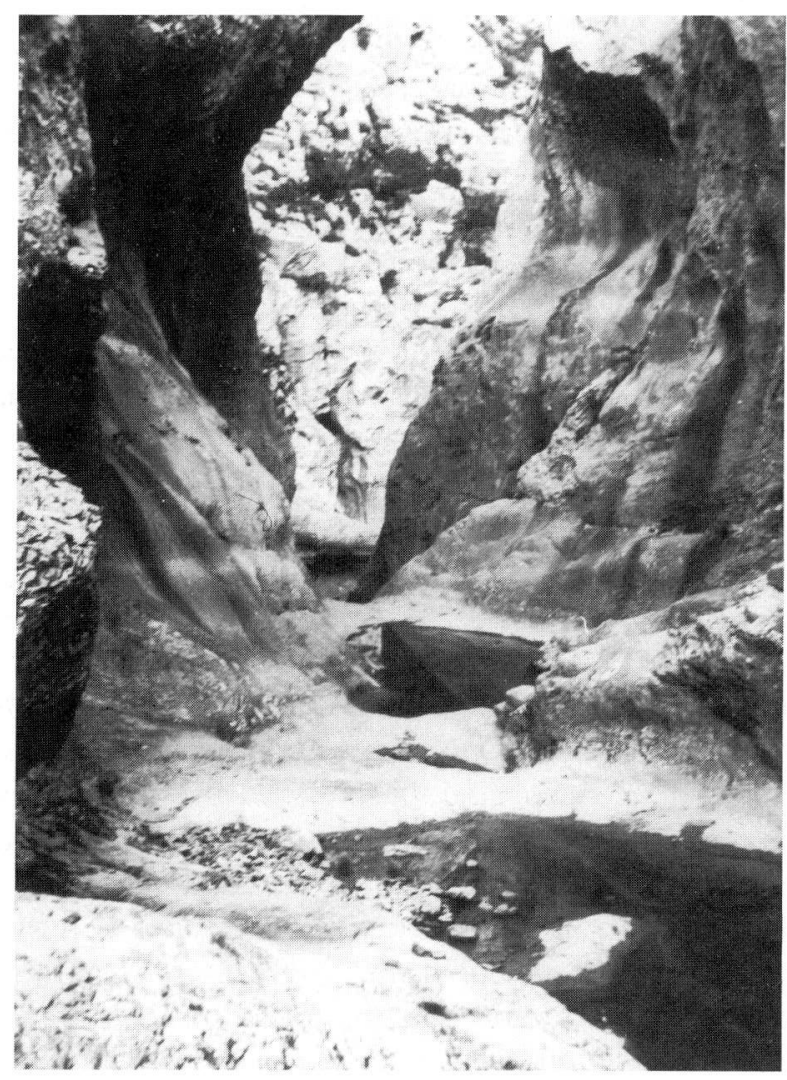




\section{LA RIPRESA MORFOGENETICA}

Si è visto dunque che l'area qui descritta viene definita da uno stretto rapporto tra fattori morfogenetici e forme.

Ciò è ovvio, ma se si analizza con relativo dettaglio tale rapporto si riscontrano affascinanti informazioni.

Approssimandoci al piede dei primi rilievi prealpini, sia in destra che in sinistra Tagliamento, possiamo osservare pressoché ovunque stretti accessi alle vallate che ne rappresentano i collettori; si tratta quasi sempre di forre, evidentemente recenti, con forme molto giovanili al contorno, almeno fino ad una certa quota dal fondovalle.

Risalendo inoltre le valli stesse, si rilevano di frequente anse e confluenze angolate e testate a " $t$ ".

Queste morfologie sono note per la loro dipendenza da fatti tettonici recenti, a meno che non esistano presupposti esclusivamente litologici, il che è però fatto più raro nella zona qui descritta.

Lavori recenti denunciano l'esistenza di movimenti, non meglio definiti se non come positivi o negativi, che evidentemente intervengono sui tempi e sulle modalità dell'evoluzione.

Poiché da essi risulta che la parte medio superiore del Friuli e il Carso sono in sollevamento, appare evidente come la rete drenante, primo tra gli agenti morfogenetici attualmente dominanti, abbia potuto e tuttora possa risentire di questa influenza adeguandovisi vistosamente.

Si giustificano allora le sovrapposizioni di forme indici di stadio diverso e soprattutto gli arretramenti di stadio morfogenetico riscontrati pressoché ovunque nei rilievi alpini e nell'area carsica (dove qui esista ancora attività della rete drenante superficiale).

Abbiamo già descritto gli esempi più vistosi delle valli alpine e prealpine e della Val Rosandra. Riteniamo che esse siano testimonianze certe del processo in atto, riconoscibili da quelle analoghe, attribuite ad altra causa (valli sospese ecc.), per il loro carattere anisotropo rispetto al contorno.

Da quanto detto possiamo allora concludere che in quasi tutta la regione è in corso un processo strutturale che attiva molte delle evidenze morfogenetiche usualmente riscontrabili nella nostra fascia morfoclimati$\mathrm{ca}$; ciò significa che le forme che rileviamo non sono un risultato finale, ma piuttosto l'inizio di un nuovo arco di ciclo evolutivo strettamente collegato con gli eventi geodinamici.

Nonostante non sia stata assolutamente chiarita l'entità di questi ultimi né l'insieme delle loro cause prime, sembra lecito concludere che la fase 
in atto sia in grado di determinare modifiche sostanziali, già percettibili o vistosamente impresse nel territorio. Tale constatazione è importante, perché in essa si precisano i motivi del paesaggio del Friuli-Venezia Giulia quale risulta oggi e quale presumibilmente si delineerà nel prossimo futuro. Ancora una volta si conferma l'impossibilità di descrivere staticamente questi diversi ambienti, senza incorrere in un errore di fondo: l'ignoranza dell'agente morfogenetico "tempo".

\section{SUMMARY}

The morphology of the Julian Alps and Prealps and of the Triestian Karst are here shortly described; the structures, which defined its origin and development, are also considered.

We can notice some cliffs which follow one another from the State boundary to the Adriatic sea; they are made up by lithologic successions, which repeat themselves according to the latitude. Anyway we recognize a general outcropping of rock masses which are decreasing little by little southward as regards the altitude, the age and than the erodibility.

There are some differences between the western (Carnian) and the eastern (Julian) bend of the regional mounts according not only to the latitude but to the longitude, because of the lithology.

In fatc, the Julian relieves often look like the Dolomities in the northern zone. The southern ones, particularly near the high alluvial plain, are sweet and gently dipping. The drainage nets are quite different too. In the upper zone it is a trellis net, in the lower one is locally a trellis net and than it becomes a dendritic system.

The glacial erosion follows the same principle too, coming southward along the main and the subordined valley cuts.

At last, the Karst morphology shows itself strongly conditioned by the structural scheme as well as by the lateral lithologic changes. It comes out an anisotropic whole of surface forms and of subsurface ones, clearly refered to those reasons.

The whole area here described shows moreover a high evolutive dinamycs, connected with the recent tectonic phases.

\section{BIBLIOGRAFIA}

AUTORI VARI 1977: Studio geologico dell'area maggiormente colpita dal terremoto friulano del 1976. Riv. It. Paleont. e Strat., 83, 2, 193-393, Milano.

Carobene L., Carulli G.B. and Vaia F. 1981: $F^{\circ}$ Udine. In: carta tettonica delle Alpi meridionali alla scala 1:200.000. A cura di A. Castellarin. P.F. Geodinamica, pubbl. 441, 39-45, Bologna. 
CARUlli G.B. AND AL. 1980: Evoluzione plio-quaternaria del Friuli e della Venezia Giulia. Contrib. prel. a. realizz. d. Carta Neotett. d'Italia. Pubbl. 356, P.F. Geodinamica, 487-523, Napoli.

CASALE A. AND VAIA F. 1972: Relazione tra schema deformativo e cavità carsiche nell'abisso "Michele Gortani". (M. Canin-Alpi Giulie). Atti e Mem. Comm. Gr. "E. Boegan', 11, 67-94, Trieste.

Croce D. AND VAIA F. 1985: Aspetti geomorfologici dell'anfiteatro tilaventino (Friuli). Gortania-Atti Mus. Friul. St. Nat., 7, 5-36, Udine.

CUCCHI F., PIRINI RAdRIZZANI C. AND PUGLIESE N. 1987: The stratigraphical sequence of the Trieste Karst. Mem. Soc. Geol. It., 40, 35-44.

Feruglio E. 1925: Le Prealpi fra l'Isonzo e l'Arzino. Boll. Ass. Agr. Friul., 7, pp. 108, Udine.

FORTI F. 1972: Proposta di una scala di carsificabilità epigea nelle carbonatiti calcaree del Carso Triestino. Atti Mus. Civ. St. Nat., 28, 3, Trieste.

GUBIANI R. AND VAIA F. 1982: Morfogenesi differenziata nei dintorni di Gemona del Friuli. Gortania-Atti Mus. Friul. St. Nat., 4, 41-64, Udine.

IACUZZI R. AND VAIA F. 1977: Studio geomorfologico dell'alto bacino del Torre (Friuli). Ed. Grillo, pp. 44, Udine.

IACUZZI R. AND VAIA F. 1981: Clima e idrogeologia nei bacini prealpini tra Magnano in Riviera a Faedis (Friuli). Gortania-Atti Mus. Friul. St. Nat., 2, 5-48, Udine.

IACUZZI R. AND VAIA F. 1981b: Carte tematiche del territorio della Comunità Montana delle Valli del Torre (Friuli). Ed. Arti Grafiche, Udine.

MARTINIS B. 1962: Ricerche geologiche e paleontologiche della regione compresa tra il $T$. Iudrio e il F. Timavo (Friuli orientale). Riv. It. Paleont. e Strat., 8, pp. 246, Milano.

MARTINIS B. 1975: The Friulian and Julian Alps and Prealps. In: Structural model of Italy. Quad. Ric. Sc., 90, Roma.

SELLI R. 1963: Schema geologico delle Alpi carniche e Giulie occidentali. Giorn. Geol., 2, 30, 1-136, Bologna.

StACHE G. 1889: Die Liburnische Stufe und deren Grenz Horizonte. Erste Abteilung. Abh. der K.K. Geol. Reichanst., 13, 1-170, Wien.

UlCIGRAI F. 1969: Prime osservazioni sui termini calcareo-dolomitici cenomaniani del Carso Triestino. Boll. Bibl. Mus. Civ. Bienn. Arte Ant., 1-7, Udine.

VAIA F. AND ZORZIN F. 1981: Fenomeni di tettonica recente in Val Resia (Prealpi Giulie). Gortania-Atti Mus. Friul. St. Nat., 3, 5-20, Udine.

VENZO G.A. AND FugANTI A. 1965: Analisi strutturale delle deformazioni tettoniche del Carso Goriziano (Gorizia). St. Trent. Sc. Nat., sez. A., 42, 2, Trento.

ZANFERRAri A. 1977: Aspetti geologici dei terremoti del 1976 in Friuli. Friuli, 6 maggio 1976. Mus. Civ. St. Nat., Trieste. 